\title{
Landkarten des Ungewissen als Werkzeug in der angewandten Zukunftsforschung
}

\author{
Authors: Hans-Liudger Dienel, Christoph Henseler \\ Submitted: $\quad$ 7. January 2020 \\ Published: $\quad$ 14. January 2020 \\ Volume: 7 \\ Issue: 1 \\ Affiliation: Technische Universität Berlin \\ Languages: \\ Keywords: \\ German \\ Maps of Uncertainties, Futurology, Science communication, \\ Inverse infographics \\ Categories: Humanities, Social Sciences and Law \\ DOI: $\quad$ 10.17160/josha.7.1.624
}

\section{Abstract:}

While uncertainty, incertitude, unknown and nescience is not only an accepted but favoured attitude within scientific debates, it is less allowed in science communication to and with society. In science communication, still affirmative messages are expected and thus delivered. The societal expectation against scientific messages obviously was and is transformed into an inner expectation of scientists against themselves. The paper - after giving an overview on the lively discussion about scientific uncertainty and nescience (landscapes of uncertainties) - presents a new tool for the communication of scientific uncertainties in "Maps of Uncertainties". These maps are a new inverse form of infographics, which shall allow a different communication of uncertainties and thus a different science-society-relation. The paper presents and discusses six exemplary maps.

\section{JOSHA Jumna ossemene. Humanities and Arts}




\title{
Landkarten des Ungewissen
}

\section{als Werkzeug in der angewandten Zukunftsforschung}

\author{
Hans-Liudger Dienel, Christoph Henseler
}

"Ich habe bemerkt", sagte Herr K., "dass wir viele abschrecken von unserer Lehre dadurch, dass wir auf alles eine Antwort wissen. Könnten wir nicht im Interesse der Propaganda eine Liste der Fragen aufstellen, die uns ganz ungelöst erscheinen?"

Berthold Brecht, Geschichten von Herrn Keuner

\begin{abstract}
Der innerwissenschaftliche Diskurs gehorcht anderen Erwartungshorizonten als die Wissenschaftskommunikation mit der Gesellschaft. Gegenüber der Gesellschaft sieht sich die Wissenschaft oft mit der Erwartung konfrontiert, nicht Fragen und Problemstellungen sondern Lösungen und affirmative Botschaften zu kommunizieren. Angesichts dieser Problemlage haben wir am Beispiel der Kommunikation wissenschaftlicher Forschungsergebnisse zu Extremereignissen ein neues Instrument für die Wissenschaftskommunikation entwickelt und getestet: die Landkarten des Ungewissen. Das sind inverse Infografiken der Ungewissheit, des Ungefähren und auch des Nichtwissens. Auf diese Weise möchten wir das Kernleistungsprofil von Wissenschaft, analytisch zu hinterfragen, für die Gesellschaft besser aufbereiten und fruchtbar machen. Der Beitrag stellt also ein neues Werkzeug für die Wissenschaftskommunikation und das Wissenschaftsmarketing dar.

Denn die oft als mäßig wahrgenommenen Erfolge wissenschaftlicher Modelle und Erklärungen von Krisen und Katastrophen sind Anlass für Unzufriedenheit und Schuldzuweisungen gegenüber der Wissenschaft und führen zu Hilflosigkeit und Fatalismus gegenüber Extremereignissen. Eines der größten Probleme in der öffentlichen Wahrnehmung von Wissenschaft ist die Überschätzung des dort tatsächlich vorhandenen Wissens bzw. der verwendeten Modelle. Während im innerwissenschaftlichen Diskurs in den einzelnen Disziplinen die Grenzen dessen, was man wirklich erkennen kann - was gewusst, was vermutet und was gänzlich ungewiss ist - meist gut bekannt sind, so gilt dies keineswegs für die Wahrnehmung in der Öffentlichkeit. Hier werden wissenschaftliche Erkenntnislagen oft weit überschätzt und hier wird, so die These, die Außenerwartung an die Wissenschaft zur Innenerwartung der Wissenschaftler/Innen an ihre gesellschaftliche Kommunikation.
\end{abstract}


Als Gegengewicht zu der bisherigen Wissenschaftskommunikation haben wir in den Landkarten des Ungewissen am Beispiel von auch im öffentlichen Diskurs stark wahrgenommenen Extremereignissen Nicht-Wissen und Ungewissheiten im gegenwärtigen Forschungsstand der jeweiligen Disziplinen recherchiert, mit Experten/innen der jeweiligen Felder diskutiert und zu prägnanten Aussagen weiterentwickelt. Diese wurden dann in einem iterativen Prozess in prägnante, inverse Infografiken (die Landkarten des Ungewissen) umgesetzt, welche die Begrenztheit vorhandener wissenschaftlicher Erkenntnisse im Bereich von Extremereignissen verdeutlichen, um eine gesellschaftliche Diskussion über den Umgang mit Nichtwissen zu fördern. Mit diesen Landkarten stellt das von der VW-Stiftung geförderte Projekt ein Werkzeug für die Wissenschaftskommunikation vor.

Der Beitrag hätte aus Volker Trommsdorffs berühmter Innovationswerkstatt an der TU Berlin stammen können, die so viele Ideen geboren hat. Ihm ist der Beitrag gewidmet.

\section{Ansatz und Vorgehen}

In seinem Buch Uncertain Science ... uncertain world stellt der Geophysiker und Klimaforscher Henry Pollack fest: "The uncertainties that scientists face are really not so different from the uncertainties we encounter in daily life. ... Ironically, people who are not scientists often equate science with certainty, rather than uncertainty" (Pollack 2003, S. 6). Mit dem Projekt "Landkarten des Ungewissen" wollten wir ein Werkzeug entwickeln, welches diese Wissenschaftswahrnehmung ändern kann.

Ziel des von der Volkswagenstiftung geförderten Forschungsvorhabens war es, als Gegengewicht $\mathrm{zu}$ der bisherigen Wissenschaftskommunikation die Begrenztheit und die Lücken des momentanen Erkenntnisstandes am Beispiel von sechs Extremereignissen auszuloten und in einem neuen Typ von inversen Infografiken (den Landkarten) zu visualisieren und kommunizierbar zu machen. Dabei sollte es ausdrücklich nicht um ein Erfassen neuer Forschungsfragen gehen, sondern darum, die inhärenten Blind spots der Disziplinen und die Beschränkungen und Umstrittenheit von Modellen und Erklärungen und Vorhersagen zu vermitteln. Diese sind in den wissenschaftlichen Disziplinen selbst gut bekannt, müssen jedoch thematisiert und vermittelt werden, um so überzogenen Erwartungshaltungen vorzubeugen und einen mündigen Umgang mit wissenschaftlichen Erkenntnissen zu fördern.

Das Beispiel der Extremereignisse war dem Förderschwerpunkt der Stiftung geschuldet;es hätte auch ein ganz anderes Beispiel bearbeiten können. Bei der Auswahl der konkreten Extremereignisse, also der Themen für die einzelnen Infografiken wurde angestrebt, (1) unterschiedliche wissenschaftliche Disziplinen (Modell- und Ingenieurwissenschaften, Sozialund Wirtschaftswissenschaften, Naturwissenschaften) und (2) Dimensionen des Nichtwissens (genuines Nichtwissen, Umstrittenes, widerstreitende Meinungen, Wahrnehmungsdifferenzen, Transferprobleme) zu verknüpfen.

Dabei ging das Projektteam von der Koexistenz epistemischer Kulturen aus (vgl. Böschen et all 2006, S. 294-301), die charakteristische Formen des Umgangs mit dem Unerwarteten und Ungewissen herausgebildet haben. Jede der entwickelten Landkarten beinhaltet damit nicht nur prägnante und überraschende Erkenntnisse zu den behandelten Extremereignissen selbst (was wissen wir? - was wissen wir nicht?), sondern vermittelt über den Einzelfall hinausreichende (generalisierbare) Botschaften zu typischen Problemen der (Nicht-)wissenskommunikation in den Wissenschaften. 


\section{Unsicherheit, Ungewissheit und Nicht-Wissen in Wissenschaft und Medien}

Die Erfahrungen moderner Gesellschaften mit anfänglich gefeierten neuen Technologien und technologischen Innovationen, die aber später scheiterten oder unerwartete und schädliche Nebenwirkungen verursachten, führten seit den 1960er Jahren zu einer zunehmenden gesellschaftlichen Aufmerksamkeit für Unsicherheit und Unwissenheit in Wissenschaft und Politik. Die implizite Annahme, dass, wenn wir nicht wissen, was passieren wird, die Entwicklung in die falsche Richtung laufen könnte, förderte die Beschäftigung mit den Themen Unsicherheit und Unwissenheit als eine zentrale Forschungsaufgabe im Gebiet von Technologiebewertung und STS (Science, Technology and Society). Eine exemplarische Rolle für die Diskussion der Nebenwirkungen wissenschaftlicher Innovationen spielte die Debatte über FCKWs (Wehling 2006, S. 83 ff.). Der Soziologe Ulrich Beck stellte in den 1980er Jahren in der Risikogesellschaft und nachfolgenden Arbeiten den gesellschaftlichen Umgang mit Unsicherheiten und Risiken in das Zentrum seines Konzepts der reflexiven Moderne (Beck 1992, 1999).

Die gewachsene Aufmerksamkeit für Unsicherheit und Ungewissheit führte auch zu einer Neubewertung ihrer Rolle in Wissenschaft und Gesellschaft. Smithson wies darauf hin, dass die Wissenschaft nicht immer dazu dient, Nichtwissen auszulöschen, sondern Nichtwissen manchmal absichtlich konstruiert wird und ganz verschiedenen Zwecken dient. (vgl. Smithson 1993 und 1989; Ravetz1987; Smithson 1985 und 1989; Wehling 2006; Proctor 2008; Wehling 2009; Wehling/Böschen 2015).

In der Wissenschaft selbst hat Unwissenheit viele positive Konnotationen. Schon Merton betonte, dass die gesellschaftlich definierte Rolle des Wissenschaftlers sowohl auf die Vermehrung des Wissens als auch auf die Spezifizierung von Unwissenheit zielt. Auch Ungewissheit dient, so Merton, dem Interesse der Wissenschaft, da sie ein Argument für neue Forschung und Finanzierung darstellt (vgl. Merton 1987, S. 22). Ein illustratives, archetypisches Beispiel für dieses Selbstverständnis liefert Schatz, der in einem kürzlich erschienenen an die breite Öffentlichkeit gerichteten Artikel über die Wissenschaft ausführt, dass es das höchste Ziel der Wissenschaft sei, neue Ungewissheiten zu schaffen, etwa zu entdecken, das wir gar nicht gewusst haben, dass wir etwas nicht gewusst haben (vgl. Schatz 2012, S. 14-18).

Aber während Ungewissheit und Unwissenheit für die Wissenschaft eine gute Sache sein könnten, muss sie für den Politikprozess in ein »nützliches Nichtwissen « verwandelt werden, vergleichbar zu nützlichem Wissen (vgl. Ravetz 1987,S. 429). Während schon die Bereitstellung von nützlichem Wissen schwierig ist, wie das Beispiel der evidenzbasierten Politik zeigt, gilt dies für nützliches Nichtwissen bzw. nützliche Ungewissheit umso mehr. Dabei kann Nichtwissen nützlich für ganz unterschiedliche wissenschaftliche Zwecke sein.

So ist die Behauptung wissenschaftlicher Ungewissheit und Unwissenheit eine bekannte und gut dokumentierte Taktik in Diskursen über Risiken, welche die wirtschaftlichen Interessen starker Interessengruppen berühren. Beispiele sind etwa die Zweifel der (Tabak)Industriean den (Krebs)Risiken des Rauchens (vgl. Proctor 1995), oder der Gentechnik (vgl. Böschen et al. 2006), der Biotechnologie (vgl. Böschen et al. 2010) oder des Mobilfunks (vgl. Stilgoe 2007; Böschen 2010) oder des Klimawandels (vgl. Oreskesua 2008,;Wilson 200; Corbet/Durfee 2004). Die absichtliche Produktion von Ungewissheit und Nichtwissen sind so bedeutend, dass 
dieses Phänomen zu einem eigenen Begriff und Studiengebiet führte, der Agnotologie (vgl. Proktor 2008).

Die Medien spielen für die Stärkung der Unsicherheit gegenüber wissenschaftlichen Aussagen oft eine verstärkende Rolle, und zwar weniger durch eine eigene explizite Agenda, sondern vielmehr unintendiert. Gerade die Neutralität der Medien und ihre professionelle Gewohnheit, konkurrierende Ansichten zu präsentieren, verschafft fast zwangsläufig Randpositionen in der Wissenschaft eine überproportionale Aufmerksamkeit, so dass in der Öffentlichkeit auch ein breiter wissenschaftlicher Konsens oft nicht korrekt wiedergegeben wird (vgl. Stocking/Holstein 2008, S. 24-25. So wird aus der traditionellen Stärke unabhängiger Medien unversehens eine Schwäche (vgl. Christensen 2008, S. 266-82). In komplexen Akteurskonstellationen, in denen politische Entscheidungsträger und organisierte Interessen aus Industrie und Zivilgesellschaft miteinander konkurrieren, dienen Medien als wichtiger Sender und Verstärker für Unwissenheit. Dieser Effekt ist auch deshalb so stark, weil Wissenschaft in der Öffentlichkeit eine zentrale Rolle einnimmt. Bei stark umstrittenen Themen verstärken die Medien also oft die Ungewissheit.

Bei der Mehrheit der Themen scheint aber das Gegenteil der Fall zu sein. So fand Lehmkuhl in einer Studie von 21 Fällen journalistischer Berichterstattung über wissenschaftliche Erkenntnisse heraus, dass die Entscheidung über die Weitergabe innerwissenschaftlicher Unsicherheit an die Gesellschaft fast ausschließlich von den Bedürfnissen der erzählten Story getragen wurde. Nur wenn die intendierte Geschichte die Unwissenheit, das Nichtwissen und offene wissenschaftliche Kontroversen brauchte, kamen letztere auch vor, ansonsten wurden sie weggelassen ( vgl. Lehmkuhl/Peters 2016, S. 898-908).

Dies spiegelt sich in anderen Erkenntnissen über wissenschaftliche Berichterstattung. Im Allgemeinen neigen die Medien dazu, die Gewissheit wissenschaftlicher Erkenntnisse zu übertreiben. Dieses Vorgehen trifft auf praktisch alle Disziplinen zu, auch etwa in der Berichterstattung über die Sozialwissenschaften, in der Weiss und Singer unter den häufigsten Fehlern die übermäßige Gewissheit und viel zu frühe Schließung der Debatte feststellten (vgl. Weiss/Singer 1988, 132ff.). Es gilt stärker noch für die Naturwissenschaften (vgl. Singer/Endreny 1993, 150ff.). Das Ausblenden innerwissenschaftlicher Ungewissheit geschieht übrigens meistens nicht durch eine aktive Fehlinterpretation, sondern vor allem durch Auslassen von Einschränkungen im Geltungsbereich der ursprünglichen wissenschaftlichen Ergebnisse (vgl. Fahnestock 1986, S. 281; Singer/Endreny 1994, S. 261-270).

Diese Situation schadet dem Ansehen der Wissenschaft. So beschreibt Parascandolan Beispielen aus der Gesundheitsforschung, wie das Weglassen von Einschränkungen mittelfristig die Glaubwürdigkeit der medizinischen Forschung und Profession untergrub. Wenn falsche Prognosen nicht eintrafen, drohte der Wissenschaft die Gefahr, sich lächerlich zu machen (vgl. Parascandola 2000, S. 27).

Demgegenüber kann der Hinweis auf Unsicherheit in den Medien auch dazu dienen, das Vertrauen in wissenschaftliche Qualitäten wieder zu stärken. Wie Zehr in einer eingehenden Analyse von Medienberichten über die globale Erwärmung zeigen konnte, dienten Hinweise auf die Unvollständigkeit von Ergebnissen und die Ungewissheit des Wissens dazu, den Unterschied zwischen einer um- und vorsichtigen Wissenschaft auf einer einen Seite und einer aktivistischen und falsch informierten Öffentlichkeit auf der anderen zu markieren (vgl. Zehr 2000, S. 94). Die glaubwürdigkeitssteigernde Wirkung der Schilderung von Ungewissheit wird auch durch eine umfassende Studie von Jensen gestützt. Er befragte 601 Teilnehmer/innen, die Informationen über wissenschaftliche Ergebnisse mit oder ohne Einschränkungen erhielten, 
und konnte zeigen, dass die Einschränkungen das Vertrauen in die wissenschaftlichen Aussagen steigerten (vgl. Jensen 2008, S. 347-69).

Zusammenfassend kann man sagen, dass es nach wie vor eine allgemeine Überschätzung wissenschaftlicher Allwissenheit und zugleich bei bestimmten Kontroversen eine Unterschätzung wissenschaftlicher Aussagefähigkeit gibt. Die Landkarten des Ungewissen zielen darauf $a b$, der Wissenschaft ein Werkzeug für die Kommunikation von Ungewissheit zur Verfügung zu stellen, welches zugleich das Vertrauen in wissenschaftliche Aussagen insgesamt erhöht.

Das Werkzeug der Landkarten nimmt dabei Bezug auf neue Design- und Publikationstechnologien in der Wissenschaft, vor allem das Web, das in den letzten Jahren auch zu einem boomartigen Anstieg der Infografiken führte. Diese integrierten visuellen Diagramme für die Kommunikation von Wissen veränderten Tuftes Forderung nach Einfachheit (vgl. Bateman et al. 2010; Borkin et al. 2013; Harrison et al. 2015). Viele Zeitungen und Zeitschriften stellen nun regelmäßig aktuelle Fakten und Zahlen in Infografiken dar, was diese zu einem fast ikonischen Instrument der Daten- und Evidenzdarstellung macht.

Um zu Themen und Karten zu gelangen, wurde vom Projektteam in einem ersten Schritt eine Liste mit Extremereignissen der letzten Jahre erstellt und darunter diejenigen ausgesucht, die hinsichtlich der zu erwartenden Nichtwissensphänomene vielversprechend erschienen. Diese wurden anschließend explorativ nachrecherchiert. Zuletzt wurde eine engere Auswahl getroffen.

Nach Erstellung der ersten drei Karten wurde dann eine Zwischenevaluation vorgenommen und eine Orientierung auf die modellorientierten Wissenschaften in den ersten drei Karten festgestellt. Daraufhin wurde beschlossen, im zweiten Projektteil bei der Themenwahl gezielt Disziplinen in den Fokus zu rücken, bei denen andere Formen des Erkenntnisgewinnes im Mittelpunkt stehen (Beobachtungen, Interpretationen, Experimente, Hermeneutik).

Jedes der gewählten Themen wurde dann in Form von Desk Research aufgearbeitet und es wurden Fragen bzw. Konzepte für den Workshop entworfen. Daran anschließend wurden in den meisten Fällen mit Expertenworkshops, in zwei Fällen auch durch individuelle Interviews die zentralen Bereiche des Nichtwissens herausgearbeitet. Diese wurden aufgearbeitet, nachrecherchiert und mit den Experten abgestimmt. Dies geschah typischerweise in mehreren Iterationen. Danach wurde von Grafikern mit den Entwürfen für die Grafiken begonnen, die in mehreren Iterationen abgestimmt und schließlich übersetzt und auf ein gemeinsames Design gebracht wurden.

\section{Fallstudien: Sechs Landkarten des Ungewissen zu Extremereignissen}

Ergebnis des Prozesses sind sechs Infografiken, die jeweils eine andere Disziplin archetypisch in den Vordergrund rücken und zugleich eine bestimmte Nichtwissenssituation zum Thema haben. In ihrer finalen Anordnung bilden sie zugleich ein Narrativ, dass die Formen des Nichtwissens in den Wissenschaften und den gesellschaftlichen Umgang damit thematisiert. Dieses ist auch in Karte 1 widergegeben, die einen Wegweiser zu den anderen Infografiken und zugleich selbst eine Landkarte des Projektes darstellt. 


\section{JOSHA}

Journal of Science, Humanities and Arts

Abbildung 1: $\quad$ Karte 1-Wege des Nichtwissens. Gewusstes Unwissen: Wie Wissenschaft, Medien und Öffentlichkeit mit dem Ungewissen umgehen, Hoffnungen und Ängste wecken

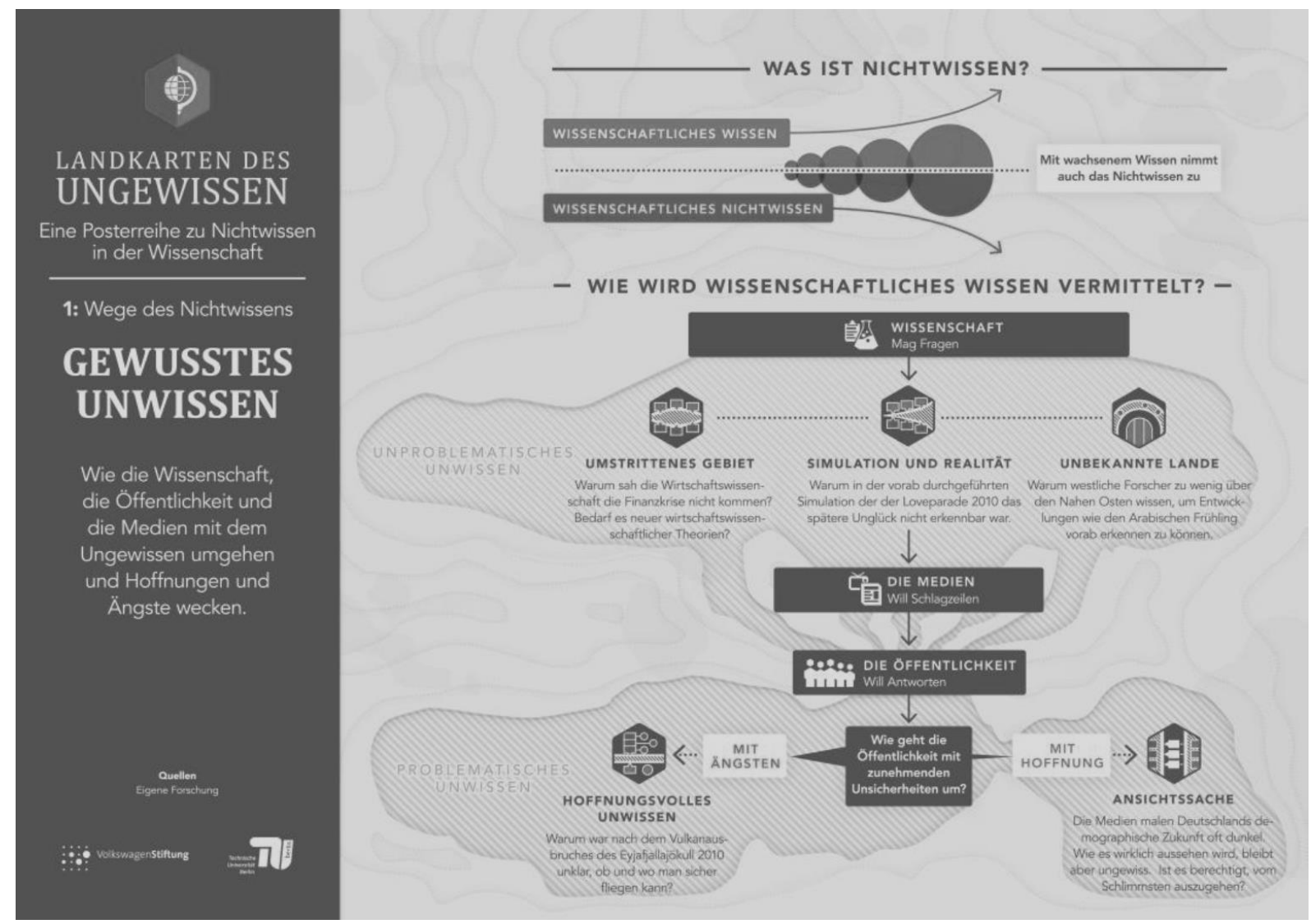

Die drei folgenden Karten widmen sich dabei archetypischen Situationen des Nichtwissens bzw. der Ungewissheit in den Wissenschaften, die gerade die Prognose extremer Ereignisse regelmäßig erschweren bzw. unmöglich machen.

Karte Zwei nimmt - am Beispiel der Wirtschaftswissenschaften und der Finanzkrise - eine der zentralen und gesunden Eigenschaften der Wissenschaften in den Blick: den Widerstreit der Meinungen im Fach und die dadurch gekennzeichneten Bereiche des Ungewissen bzw. noch nicht Gewussten in der Disziplin.

Die Herausforderung aber zugleich auch der Antrieb aller wissenschaftlichen Erkenntnis, nämlich noch nicht vorhandenes bzw. erreichbares Wissen, wird in der dritten Karte am Beispiel des westlichen Wissens über die Ursachen des arabischen Frühlings thematisiert.

Die vierte Karte widmet sich den grundsätzlichen Problemen jeder modellbasierten Voraussage: der Diskrepanz zwischen Simulation und Realität und der enormen Abhängigkeit von den gewählten Eingabegrößen. Dies geschieht am unerwarteten Beispiel der Entfluchtungsanalyse für die verhängnisvolle Loveparade in Duisburg.

Die weiteren Karten haben den zweiten größeren Aspekt des Projektes stärker zum Thema: den gesellschaftlichen Umgang mit Nichtwissen und Ungewissen. 
Dabei nimmt die fünfte Karte am Beispiel der Sperrung des europäischen Luftraumes nach dem Ausbruch des Eyjafjallajökull den typischen Umgang mit offen kommunizierten Ungewissheiten aus der Wissenschaft in den Blick. Diese werden ausschließlich als Gefahr gesehen und in klare binäre/dychatome Strukturen (fliegen/nicht fliegen) umgesetzt, um somit den aktiven Umgang mit unklaren Wissensständen zu vermeiden.

Die sechste und letzte Karte setzt diesem Bild eine Alternative entgegen. Am Beispiel des demographischen Wandels in Deutschland werden die Ungewissheiten über die Implikationen dieser Entwicklung thematisiert und den üblichen Schreckensszenarien auch positive Zukunftsentwürfe entgegengestellt. Die Karte schließt damit zugleich mit der Botschaft, dass es selbst bei sicherer Fakten- und Wissenslage immer noch der aktiven Deutung dieser Fakten bedarf.

Die Karten liegen in Deutsch und Englisch in offenen Formaten vor und eignen sich sowohl für den Einsatz in großflächigem Print, in Magazinen als auch für die online Darstellung. Mit der Wahl des Mediums (Infografiken) und der Thematik (Nichtwissen in den Wissenschaften) eignen sie sich besonders, die wissenschaftsferne Bevölkerung, die eine heterogene Zielgruppe mit kaum differenzierbaren Präferenzen und Vorverständnis darstellt, anzusprechen. Die Karten wurden und können weiter verwendet werden für Ausstellungen in Science-Centern und bürgernahen Veranstaltungen sowie zum Aushängen der Infografiken an öffentlichen Plätzen. Sie regen so einen öffentlichen Dialog für den Austausch von Wissenschaft und Öffentlichkeit an. 
Tabelle. 1: Sechs Infografiken zur Kommunikation von Ungewissheit in den Wissenschaften (am Beispiel zur Wissenschaftskommunikation von fünf Extremereignissen)

\begin{tabular}{|l|l|l|l|}
\hline \multicolumn{2}{|l|}{ Thema } & Inhalt & Disziplin(en) \\
\hline $\begin{array}{l}\text { Landkarte des } \\
\text { Ungewissen }\end{array}$ & Überblick & $\begin{array}{l}\text { Welche Ungewissheiten gibt es in } \\
\text { den Wissenschaften und wie } \\
\text { gehen wir damit um }\end{array}$ & $\begin{array}{l}\text { Wissenschaftskom- } \\
\text { munikation }\end{array}$ \\
\hline $\begin{array}{l}\text { Weltweite } \\
\text { Finanzkrise }\end{array}$ & $\begin{array}{l}\text { Umstrittenes } \\
\text { Wissen }\end{array}$ & $\begin{array}{l}\text { Konkurrierende und } \\
\text { Koexistierende Wahrheiten. } \\
\text { (Unterschiede in } \\
\text { Annahmen als Indikatoren für } \\
\text { Ungewissheitsfelder) }\end{array}$ & Wirtschaftswissenschaften \\
\hline $\begin{array}{l}\text { Love Parade } \\
\text { Duisburg2010 }\end{array}$ & $\begin{array}{l}\text { Simulation und } \\
\text { Realität }\end{array}$ & $\begin{array}{l}\text { Mangelnde Passgenauigkeit von } \\
\text { Modellen (Modell vs. „Realität) } \\
\text { und Begrenztheit von Modelle }\end{array}$ & $\begin{array}{l}\text { Simulation und } \\
\text { Modellierung generell }\end{array}$ \\
\hline $\begin{array}{l}\text { Arabischer } \\
\text { Frühling }\end{array}$ & Terra Incognita & $\begin{array}{l}\text { Erkenntnisgrenzen über andere } \\
\text { Länder aus } \\
\text { Kommunikationsmangel und - } \\
\text { unmöglichkeit }\end{array}$ & $\begin{array}{l}\text { Politik- und Sozialwissen- } \\
\text { schaften } \\
\text { (Transformations- und } \\
\text { Demokratisierungs- } \\
\text { forschung) }\end{array}$ \\
\hline $\begin{array}{l}\text { Der Ausbruch des } \\
\text { Eyjafjallajökull }\end{array}$ & $\begin{array}{l}\text { Ungewissheit } \\
\text { als Gefahr }\end{array}$ & $\begin{array}{l}\text { „Binarisierung komplexer } \\
\text { Informationen von den } \\
\text { Fachdisziplinen zu den } \\
\text { Entscheidungsträgern/ zu der } \\
\text { Öffentlichkeit }\end{array}$ & $\begin{array}{l}\text { Naturwissenschaften } \\
\text { (Meteorologie, } \\
\text { Vulkanforschung) }\end{array}$ \\
\hline $\begin{array}{l}\text { Demografischer } \\
\text { Wandel }\end{array}$ & $\begin{array}{l}\text { Ungewissheit } \\
\text { als Chance? }\end{array}$ & $\begin{array}{l}\text { Gewissheit von Daten vs. } \\
\text { Ungewissheit der Deutung }\end{array}$ & Sozialwissenschaften \\
\hline
\end{tabular}

(eigene Darstellung) 
Abbildung 2: $\quad$ Karte 2-Crashkurs: Die Weltweite Finanzkrise ${ }^{1}$
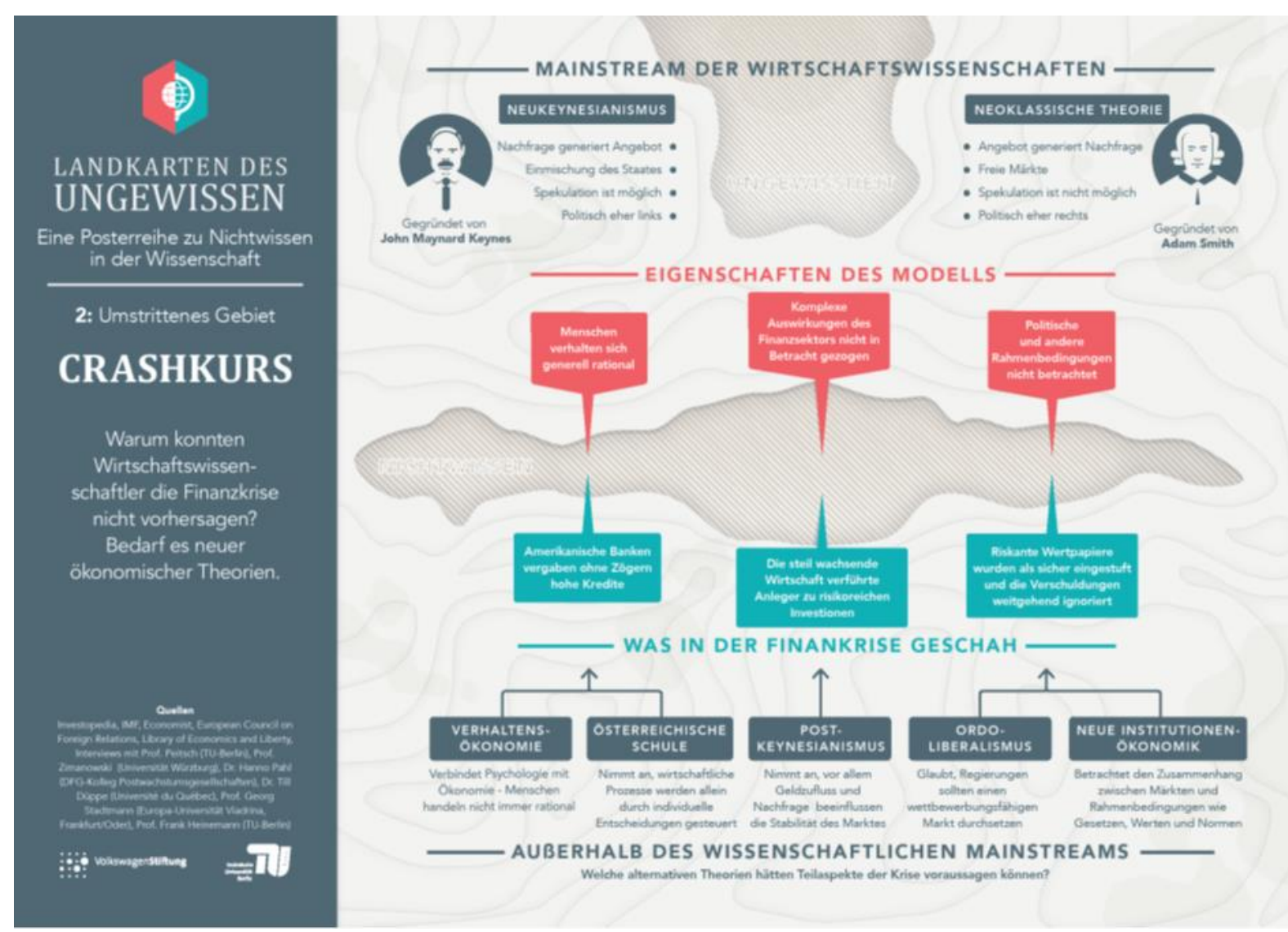

Thema: Mit dem Ausbruch der weltweiten Finanzkrise im Jahre 2008 gerieten auch die gängigen wirtschaftswissenschaftlichen Modelle und Erklärungsinstrumentarien auf den öffentlichen Prüfstand. Warum haben uns die Wirtschaftswissenschaften nicht gewarnt, geschweige denn adäquate Lösungsansätze bereitstellen können? Es scheint, dass die massiven Meinungsdifferenzen, die innerhalb der volkswirtschaftlichen Lehre ausgefochten werden, bis zu dem Ausbruch der Krise weitgehend unbemerkt an der öffentlichen Debatte vorbei gegangen sind.

Inhalt: Die Infografik bildet eine Landkarte zugleich der volkswirtschaftlichen Hauptströmungen als auch über die Meinungsunterschiede und Streitpunkte sowie Blind Spots der Wirtschaftswissenschaften.

Im Mittelpunkt stehen dabei die Meinungsunterschiede zwischen den beiden Schulen, die den volkswirtschaftlichen Mainstream bilden, das Fach prägen und ihre Fundierung zum einen auf dem DSGE-Modell (Dynamic Stochastic General Equilibrium) und den sogenannten heterodoxen Schulen auf der anderen Seite haben. Denn genau diese Punkte des Dissenses

1 Vielen Dank an Prof. Dieter Peitsch (TU-Berlin) Prof. Bernd Zimanowski (Universität Würzburg), Dr. Hanno Pahl (DFG-Forschungskolleg "Postwachstumsgesellschaften"), Dr. Till Düppe (Université du Québec), Prof. Georg Stadtmann (Europa-Universität Viadrina, Frankfurt/Oder) und Prof. Frank Heinemann(TU Berlin) für Information und Beratung zum Kartenthema. 


\section{JOSHA}

Journal of Science, Humanities and Arts

offenbaren zugleich die zentralen Blind spots des volkswirtschaftlichen Mainstreams, der gerade die wichtigsten Faktoren für die Krise in seinen Modellen nicht betrachtete. Als Ergebnis lässt sich festhalten, dass das Nichtwissen in Bezug auf die Finanzkrise vor allem endogen (in den Wirtschaftswissenschaften selbst) erzeugt wurde.

Die meisten Modelle betrachten die auslösenden Faktoren kaum, so dass in der Prognose krisenhafter Marktsituationen erheblicher Nachholbedarf besteht.

Abbildung 3: $\quad$ Karte 3-Der Arabische Frühling ${ }^{2}$

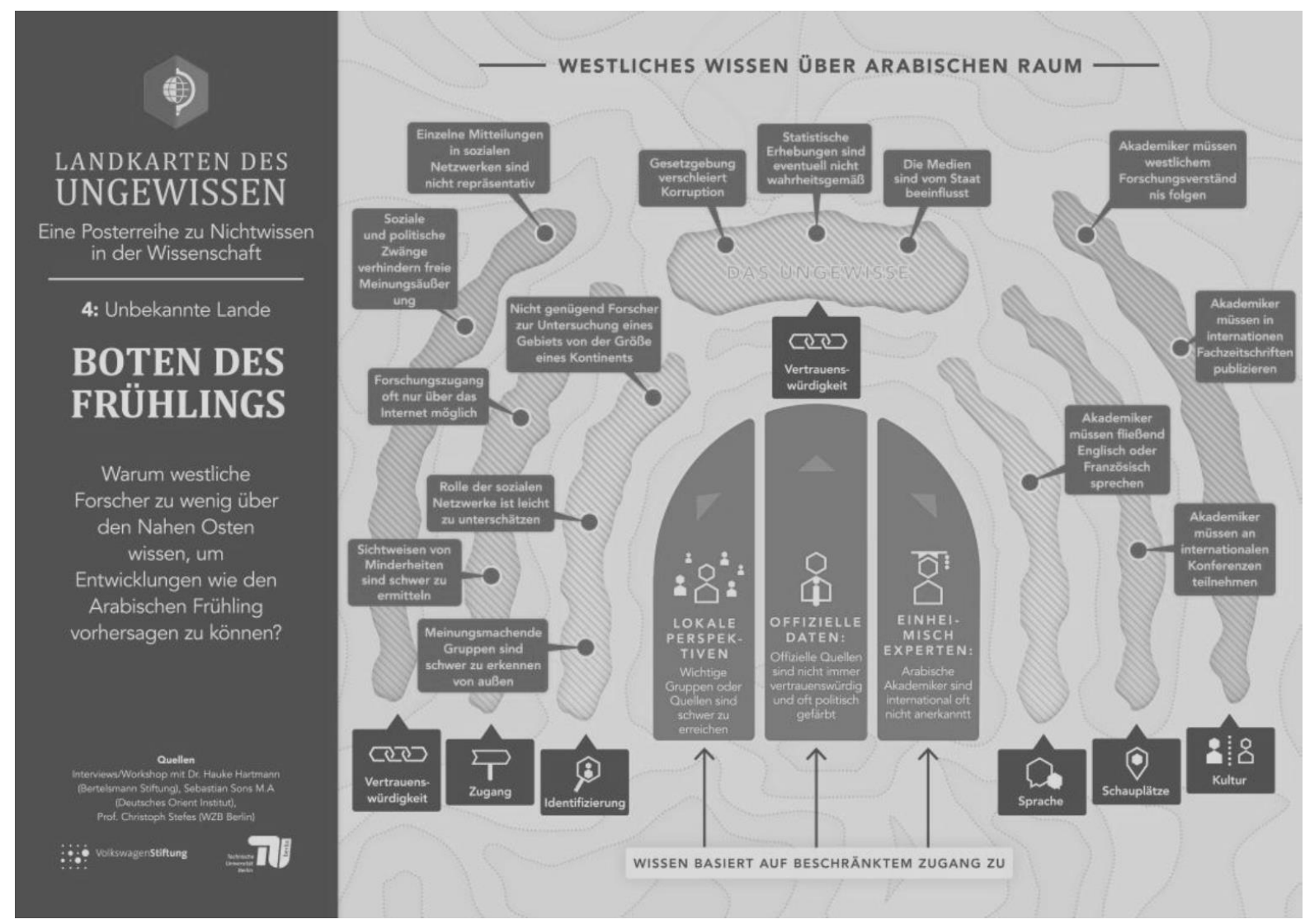

Thema: 2010/11: Massenproteste in Teilen des Nahen Ostens und Nordafrikas fordern Würde, Freiheit und Gerechtigkeit. Langjährige autokratische Systeme destabilisieren sich und in verschiedenen Staaten beginnen Bürgerrevolten. Die Verläufe und Ausmaße der Aufstände weisen regionale Unterschiede auf: Während in Ägypten, Tunesien, Libyen und Jemen ein derzeit ergebnisoffener Transformationsprozess stattfindet, beobachtet man in Syrien Bürgerund Stellvertreterkriege. In Bahrein werden die Protestbewegungen mit Hilfe der Golfkooperationsstaaten unterdrückt und auch in anderen Staaten der Region steigt der öffentliche Druck und die Protest (Bemühungen).

Doch weder die großflächigen Proteste und Demokratiebewegungen noch die inzwischen erfolgten Konterrevolutionen wurden von Experten vorausgesehen. Wie konnte dies trotz des hohen Interesses am Nahen Osten so unbemerkt bleiben?

2 Vielen Dank an Dr. Hauke Hartmann (Bertelsmann Stiftung), Sebastian Sons M.A (Deutsches Orient Institut) und Prof. Christoph Stefes (WZB Berlin) für Informationen und Beratung zum Thema der Karte. 
Inhalt: Im Austausch mit Wissenschaftlern verschiedener disziplinärer Hintergründe (Länderkunde, quantitative internationale Politikforschung, Indexentwicklung), dass das Hauptproblem hier tatsächlich im Mangel an Wissen, genauer am Zugang dazu fehlt.

Dies ist besonders deutlich bei der Identifikation und Ansprache relevanter Akteure vor Ort. Als Wissenschaftler von außen ist es schwer, überhaupt die relevanten Akteure (1) zu identifizieren und dann auch (2) Zugang zu ihnen zu finden. Zuletzt kann (3) aufgrund des repressiven Charakter der Regime und der Interaktion mit einem Außenstehenden nicht davon ausgegangen werden, dass diese sich offen äußern. Es besteht also ein signifikantes Zugangsproblem.

Dieses Problem stellt sich auch wissenschaftlichen Diskurs selbst. Die regionalen/ einheimischen Forscher, die über das meiste Wissen vor Ort verfügen, haben kaum Zugang zur internationalen Scientific Community. Die Ursachen dafür liegen in Sprachbarrieren und ,kulturellen' Unterschieden. Dies führt zu einem geringen Austausch zwischen ausländischen Wissenschaftlern und ihren Pendants vor Ort, so dass die Akteure mit dem meisten Wissen nicht international publizieren und die Publikationen von Personen mit geringerer Kenntnis der Lage vor Ort stammen.

Zuletzt können auch Methoden der Dokumenten- und Datenanalyse nur begrenzt verwendet werden, da in der Region dafür meist die notwendige Politiktransparenz fehlt, so dass offizielle Dokumente (Verfassung, Gesetze, ...) oft nicht der Umsetzung entsprechen und bei Statistiken von deren Unzuverlässigkeit ausgegangen werden muss.

Diese dreifache Zugangsbarriere westlicher Forscher zu dem zur Beurteilung der Lage und von Entwicklungen notwendigen lokalen Wissen, bildet die Karte durch eine dreifache Grabenlandschaft zwischen dem Betrachter und dem Wissen ab.

Thema: Die Love Parade fand 2010 erstmals auf einem geschlossenen Gelände statt. Das Gelände, der ehemalige Duisburger Güterbahnhof, konnte für die Besucher nur mittels eines Straßentunnels erreicht werden. Auf der Rampe vor diesem Tunnel kam es noch während der Befüllung zu einer Stauung, in dessen Folge 21 Menschen starben. Um die Chance auf eine erfolgreiche Genehmigung $\mathrm{zu}$ erhöhen, gab der Veranstalter im Vorfeld eine Entfluchtungsanalyse in Auftrag. Sie sollte zeigen, dass sich das Gelände und besonders kritische Flächen in einer akzeptablen Zeit räumen lassen - und konnte dies auch tun, so dass die Genehmigung trotz Nicht-Einhaltung vorgeschriebener Ein- und Ausgangsbreiten erteilt wurde. Die Ersteller der Analyse gerieten direkt nach der Katastrophe unter massiven Rechtfertigungsdruck. Die Kritik nahm erst ab, als im weiteren Verlauf der Diskussion der genaue Kontext des Gutachtens publik wurde.

Inhalt: Am Beispiel der Entfluchtungsanalyse zur Love Parade wird die prinzipielle Begrenztheit von Simulationen und ihre starke Abhängigkeit von den zugrunde gelegten Annahmen offenbar. Diese Grenzen werden innerhalb der Modellbildenden Wissenschaften in der Regel sehr offen kommuniziert (so auch in unserer Beispielstudie). Auf dem Weg in die Öffentlichkeit geht das „Wissen um das Nichtwissen“ jedoch häufig verloren. 


\section{JOSHA}

Journal of Science, Humanities and Arts

Abbildung 4: $\quad$ Karte 4-Liebe und Tod: Die Duisburger Loveparade ${ }^{3}$

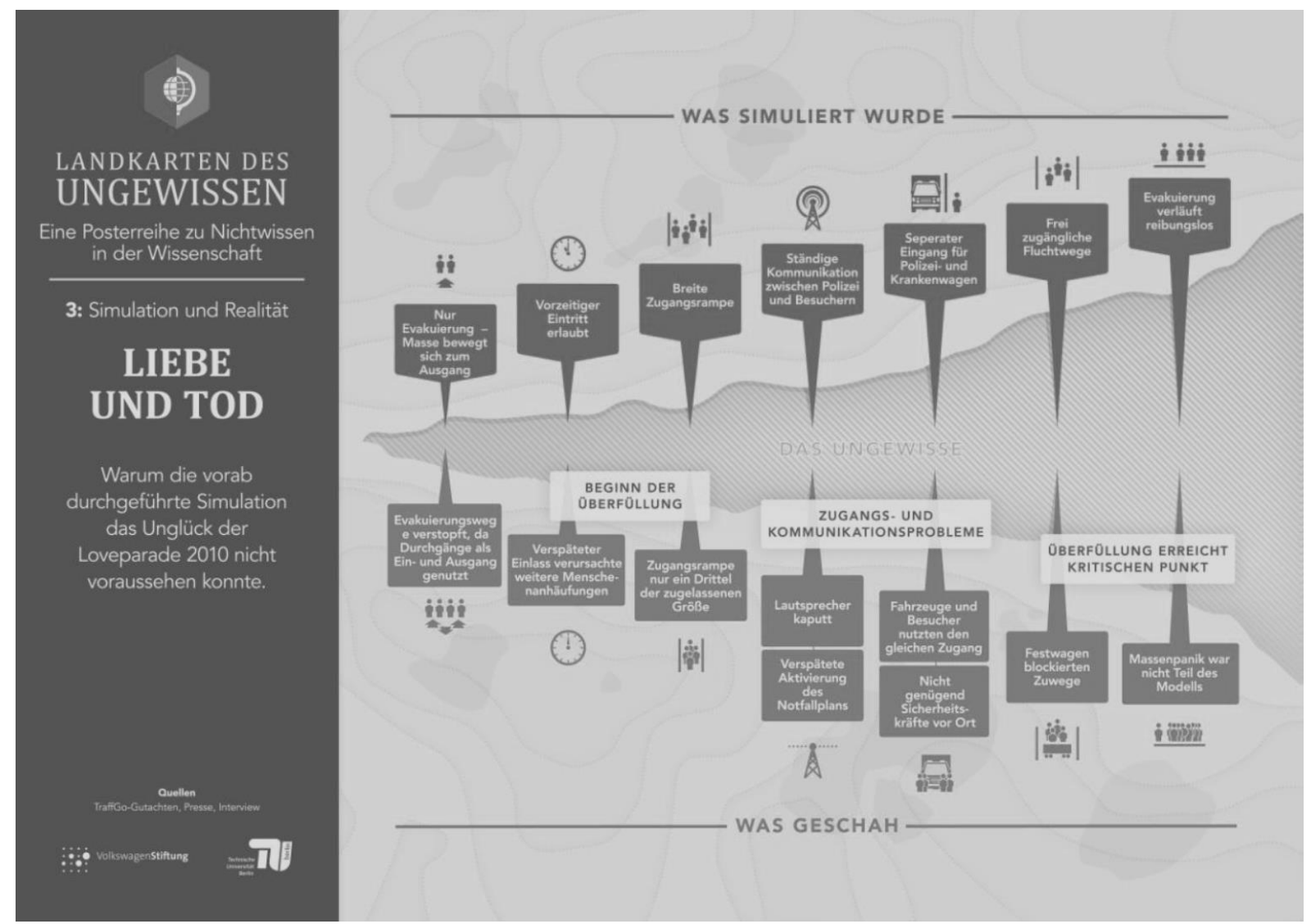

In diesem speziellen Fall lässt sich das besonders an der großen Diskrepanz zwischen den in der Simulation getroffenen Annahmen und der real stattgefunden Situation verdeutlichen. Dies war gleich doppelt ungeeignet, um das später stattfinde Unglück zu prognostizieren: (1) Sie beschränkte sich auf wenige Szenarien und setzte dabei den falschen Schwerpunkt (Evakuierung statt regulärer Be- und Entfüllung) und sie beinhaltete (2) Annahmen, die in der Realität nicht zutrafen (z.B. kontinuierliche Besucherstromsteuerung, frühes Öffnen des Geländes).

Diese Diskrepanz zwischen Eingabegrößen des Modells und Wirklichkeit und die sich daraus ergebende Kluft zwischen Prognose und Ereignis ist ein archetypisches und oft anzutreffendes Nichtwissensphänomen und wird in der Infografik als sich immer weiteres Auseinanderklaffen sinnbildlich gemacht.

3 Vielen Dank an Dr. Hugo Klüpfel (TraffGo) für Informationen und Beratung zum Kartenthema. 


\section{JOSHA}

Journal of Science, Humanities and Arts

Abbildung 5: $\quad$ Karte 5-Der Ausbruch des Eyjafjallajökull und die Sperrung des Europäischen Luftraums ${ }^{4}$

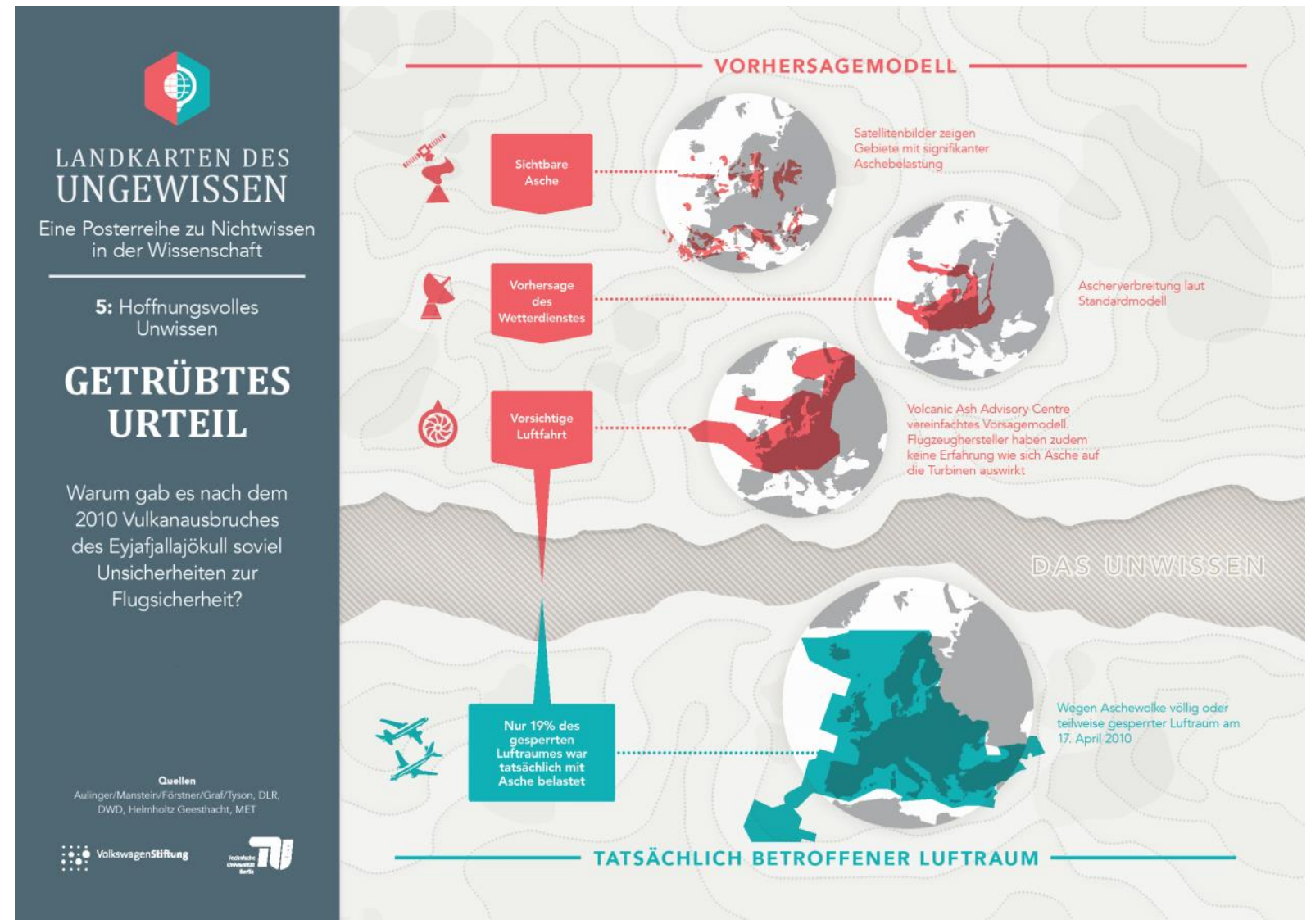

Thema: Die Eruption des isländischen Vulkans Eyjafjallajökull im Frühjahr 2010 erzeugte eine Aschewolke, die $\mathrm{zu}$ den weitreichendsten europäischen Flugausfällen seit Ende des 2.Weltkriegs führte. Obwohl ein grundsätzliches Bewusstsein für das Sicherheitsrisiko „Vulkanasche \& Luftfahrt" aufgrund vergangener Zwischenfälle vorhanden war, führte die Entscheidung weiträumiger Luftraumsperrungen $\mathrm{zu}$ heftiger Kritik durch Medien, Luftfahrtbetreiber und Branchenverbände. Das chaotisch und sprunghaft anmutende Krisenmanagement belastete jedoch auch das Vertrauen in die an der Risikoabschätzung beteiligten wissenschaftlichen Disziplinen.

Inhalt: In der fünften Karte sind die Problematik des Wissenstransfers und der gesellschaftliche Umgang mit Ungewissheiten zentraler Inhalt. Das Wissen um die Ausbreitung vulkanischer Spurenstoffe war in den Fachdisziplinen (Messtechnik, Modellierung, Fernerkundung) bereits 2010 hoch entwickelt, doch die vorhandenen komplexen Informationen waren kaum auf die hoch standardisierten Kommunikationstechniken und Entscheidungsprozesse der Luftfahrtbranche übertragbar. Kontrollbehörden und Entscheidungsträger griffen stattdessen

$4 \quad$ Vielen Dank an Dr. Armin Aulinger (Helmholtz-Zentrum Geesthacht), Dr. Hermann Manstein (Deutsches Zentrum für Luft- und Raumfahrt - DLR), Dr. Jochen Förstner (Deutscher Wetterdienst - DWD), Kaspar Graf (Deutscher Wetterdienst - DWD), Chris Tyson (Met Office) für Informationen und Beratung zuKartenthema. 


\section{JOSHA}

Journal of Science, Humanities and Arts

auf eine simplifizierte Form der Informationsgewinnung (und -kommunikation) zu Vulkanasche zurück, die lediglich grobe Polygone mit binärem Informationsgehalt vermittelten. So wurde aus den auf den Satellitenbildern erkennbaren kleinen Aschwolken eine Europaweite Luftsperrung.

Die Karte macht diese Zunahme an Nichtwissen von realen Daten bis hin zu groben Luftraumsperrungen durch die Überlagerung von (1) Echtzeit-Satellitenbild, (2) dem graphischen Output eines Dispersionsmodells, (3) der Daten der Volcanic Ash Advisory und (4) der Karte der Luftraumsperrungen anschaulich und prägnant.

Abbildung 6: $\quad$ Karte 6-Der Demografische Wandel in Deutschland

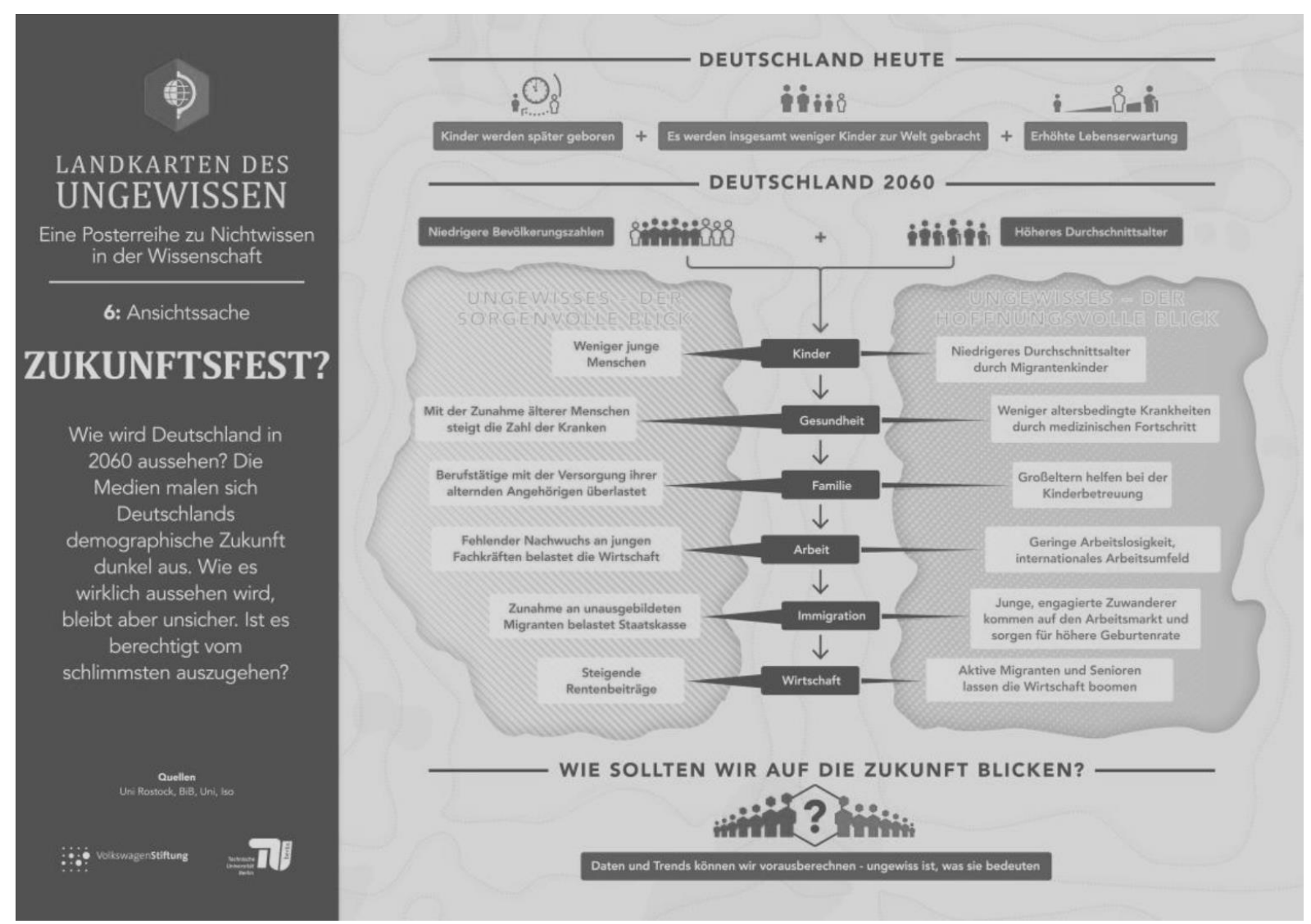

Das Beispiel: Der Begriff Demografischer Wandel ist seit Jahren ein vielseitig diskutiertes Phänomen. Beinahe jede Wissenschaftsdisziplin beschäftigt sich in irgendeiner Weise damit, die Politik hat ihn auf verschiedenen Agenden stehen, für unternehmerische Probleme muss er einstehen, die Medien greifen ihn regelmäßig als Schwerpunktthema auf. Doch unterschiedliche Akteure haben verschiedene Interessen daran, was in den Augen der Bevölkerung zu ,,verwaschenen“ und nebulösen Diskussionen führt. Was ist der Demografische Wandel und welche Konsequenzen bringt er mit sich? In Anbetracht der relevanten Diskurse zu diesem Thema erscheint der Demografische Wandel weniger als ein Megatrend als eher ein Extremereignis mit Ankündigung. 
Das Thema: Der Begriff des Demografischen Wandels meint vor allem die strukturelle und zahlenmäßige Veränderung der Zusammensetzung der Bevölkerung. In Deutschland geht es dabei in erster Linie um eine ändernde Altersstruktur sowie $\mathrm{Zu}$ - und Wegzüge in und aus bestimmten Regionen innerhalb Deutschlands als auch dem Ausland. Prägend für den demografischen Wandel in Deutschland ist die Zunahme der Älteren (ab ca. 60 Jahren) im Gegensatz zu den unter 30-jährigen. Trotz einer höheren Sterberate als Geburtenrate hält dieses Phänomen an. Im Zuge der Weiterentwicklung und kontinuierlichen Verbesserung der Gesundheitsversorgung und anderen Lebensumständen steigt die Lebenserwartung stetig an.

Das mag sicher an den vielen Facetten und unterschiedlichen Akteursinteressen liegen. Regelmäßig ist der Begriff Demografischer Wandel in der Öffentlichkeit, als auch in Politik, Wissenschaft und Wirtschaft negativ konnotiert. Der demografische Wandel wird als Bedrohung und unbedingt aufzuhaltende Entwicklung angesehen.

Doch tatsächlich können über viele Folgen des demographischen Wandels noch gar keine Aussagen getroffen werden, denn bis zum heutigen Zeitpunkt gab es in der Menschheitsgeschichte eine Gesellschaft mit einem solchen demographischen Profil. Vielmehr sind die Wirkungen dieser Veränderungen auf die gesellschaftliche Wirklichkeit schlicht: unbekannt und lassen auch alternative, positivere Deutungen zu. Diese nimmt die Karte 6 in den Blick und stellt sie am Beispiel der Themenfelder Kinder, Gesundheit, Familie; Arbeit, Migration Wirtschaft den üblichen Deutungen entgegen und schließt damit die Kartenserie über Katastrophen, Extremereignisse und Ungewisses mit einer positiven Note.

\section{$4 \quad$ Rückblick auf die Arbeit an den Landkarten und Blick nach vorn}

Bei der Arbeit an der Schnittstelle zwischen der Öffentlichkeit und ganz verschiedenen Fachdisziplinen, denen die Mitglieder der Forschungsgruppe selbst nicht unmittelbar entstammen, bleiben unerwartete Probleme, inhaltliche Abweichungen und organisatorische Hürden nicht aus, die das Projektteam zu einer Überarbeitung der ursprünglichen Planungen zwangen. Dabei erwiesen sich insbesondere zwei Aspekte als folgenschwer für den Ablauf des Projektes:

Erstens erwies sich der Aufwand für die Recherche als weit höher denn angenommen. Dies liegt darin begründet, dass in der Projektkonzeption die Erfassung der Themenfelder und Konzeption der Karteninhalte als partizipativer Prozess mit Experten geplant war, der eine geringere eigene Recherche vorsah. Allerdings stellte sich heraus, dass nicht nur für die Findung und Auswahl geeigneter Themen und für die inhaltliche Vorbereitung der Workshops und Interviews Vorstudien notwendig waren, sondern auch und gerade im Nachgang der Experteninputs noch aufwendige Recherchen und Abstimmungsarbeiten notwendig waren. Diese bestanden daraus, die von den Experten erarbeiteten Themen nachzurecherchieren sowie Zahlen- und Datenmaterial zu beschaffen und die Themen auszuarbeiten.

Darüber hinaus musste aus den in den Workshop erarbeiteten Themenfeldern des Nichtwissens eine Auswahl des Hauptthemas getroffen und dann in Hinsicht auf Visualisierund Kommunizierbarkeit mit dem Grafikbüro abgestimmt werden. Daraus ergaben sich oft weitere notwendige Recherchen und Datenbeschaffungen, die nun wiederum für die Erstellung der Grafik für das gewünschte Themenfeld nötig waren. Dieses iterative Vorgehen bedeute unter anderem auch, dass ein Vielfaches an Beispielen für Nichtwissen und Ungewisses identifiziert, Themenfelder recherchiert und Datenmaterial zusammengetragen wurden, als später in den Grafiken Verwendung fand. 
Zweitens stellte es sich dabei als besondere Herausforderung dar, dass das Projekt in Zusammenarbeit mit einem Londoner Grafikbüro (Information IsBeautiful) durchgeführt wurde. Diese Auswahl wurde aufgrund des innovativen Ansatzes schon im initialen Angebot und der besonderen Stellung, die das Studio und ihr Gründer im Bereich der Infografiken einnimmt getroffen und erwies sich hinsichtlich der Qualität der Grafiken und auch der inhaltlichen Inputs zur Zuspitzung als gut und richtig. Allerdings brachte dies auch aufwendigere Wege der Abstimmung mit sich. Dabei war die Sprachbarriere selbst das geringere Problem, sondern eher die damit verbundenen Verzögerungen, da sowohl Themen wie Workshopergebnisse und Konzeptpapiere erst übersetzt und dann die Grafiken wieder zurückübersetzt werden mussten. Diese Verzögerungen, gepaart damit, dass in einer solchen Interaktion bestimmte Nuancen verloren gingen, sorgten dafür, dass zwischen Themenfindung und Ausarbeitung sowie Finalisierung der Landkarten mehrere Monate lagen. Zuletzt machte die räumliche Entfernung eine niedrigschwellige Zusammenarbeit schwieriger und Arrangements wie die direkte Teilnahme der Grafiker an den Workshops nicht möglich. Nicht bedacht bei der Konzeption des Projektes war zudem, dass die angedachte schrittweise Erstellung und Veröffentlichung der Karten über den Projektzeitraum so nicht machbar war, da die Nichtwissensproblematik für ein konkretes Beispiel nicht im Vorhinein absehbar war, zugleich aber bestimmte Aspekte, Designs und Formelement der Grafiken sich erst in der Gesamtschau festlegen ließen. So konnte die Finalisierung der Karten nicht vor der Konzeptionierung aller Karten erfolgen. Das Zusammenspiel dieser Faktoren führte aber auch dazu, dass das Projekt sowohl einen höheren Personalaufwand wie auch eine längere Laufzeit als ursprünglich angenommen erforderte - aber eben auch qualitativ sehr hochwertige Grafiken produzierte, die zudem zweisprachig in Englisch und Deutsch vorliegen.

Herausforderung Format Infografik: eine zentrale Herausforderung des Projektes lag auch darin, die hohe Komplexität der Themen (die jeweiligen Extremereignisse) mit der angestrebten Einfachheit der Grafik zu verbinden. Dies lag vor allem darin begründet, dass jede Thematisierung und Darstellung der konkreten Nichtwissenssituation eine Erläuterung des Kontextes voraussetzt. So konkurrierte die Darstellung des Nichtwissensphänomens immer mit der des Extremereignisses selbst.

Herausforderung Thema Extremereignis: auch wenn Extremereignisse sich wegen ihrer hohen Öffentlichkeitswirksamkeit auf den ersten Blick sehr gut für die Thematisierung von Nichtwissensphänomenen in der Wissenschaft eignen, so bringt diese Wahl doch auch Probleme mit sich. Dabei ist einerseits die oben schon erwähnte Notwendigkeit, das Ereignis selbst noch einmal darzustellen und zu erläutern, zu nennen. Darüber hinaus stellte sich bei den Recherchen bzw. in den Expertengesprächen oft heraus, dass die interessanteren Aspekte oft eher in den Konstellationen zu finden sind, die bestimmtes Nichtwissen bewusst erzeugen (Agnotology) bzw. im Umgang der Entscheidungsträger mit den wissenschaftlichen Aussagen liegen. Diese liefen allerdings dem Anspruch des Projektes (keine Schuldzuweisungen, Fokus auf Wissenschaft) entgegen.

Obgleich nicht von einem größeren, multidisziplinären Konsortium durchgeführt, war das Projekt Landkarten des Ungewissen schon von der Anlage her in höchstem Maße interdisziplinär. Dies spiegelt sich schon in den Themen der im Projekt erstellten Karten wider, die jeweils eine andere Disziplin in den Fokus nahmen und die Bereiche aus Meteorologie, Vulkanologie, Aviation, Wirtschaftswissenschaften, Politologie, Länderkunde und Demographie berührten und zum Thema hatten. Dies erfordert auch eine hohe interdisziplinäre Zusammenarbeit im Projektteam selbst als auch mit Vielzahl externer Experten und Partner 
ohne die das Projekt nicht durchzuführen gewesen wäre. Man könnte die Informationsgewinnung aus und zwischen den Disziplinen sogar als eine der definierenden Eigenschaften von LdU ansehen. Auffallend ist das durchweg positive Feedback - von allen angesprochenen Wissenschaftlern und unabhängig von der fachlichen Herkunft - zu Idee und Grundprämissen des Projektes. Die Thematisierung eigener Ungewissheit und Nichtwissens ist offenbar selbst ein interdisziplinäres Problem.

Die Ansätze, Erkenntnisse und Ergebnisse des Projektes haben eine hohe Anschlussfähigkeit, wie sich schon in den ausgesprochen positiven Reaktionen der befragten/teilnehmenden Experten während des Projektes zeigte. Die Notwendigkeit, die Grenzen des eigenen Wissens und Nicht-Expertenseins zu kommunizieren, besteht über alle Wissenschaften hinweg und hat gerade in sensiblen Bereichen auch eine kritische Bedeutung.

Das Projekt Landkarten des Ungewissen dient der Kommunikation aus der Forschung und Wissenschaft in die Öffentlichkeit. Die Aufklärung/Information der Bevölkerung über Grenzen und Ungewissheiten in den Wissenschaften dient letztlich der transparenten Darlegung wissenschaftlicher und methodischer Grenzen und trägt somit zur Legitimierung der Wissenschaft bei. Die Verknüpfung mit Extremereignissen aus der jüngeren Vergangenheit soll das Interesse aller wecken. Extremereignisse aus jüngerer Vergangenheit sind in den Köpfen der Bevölkerung noch frisch und ein Bezug und Interesse ist leicht herzustellen. Bei der Auswahl der Themen wurde darauf geachtet, für verschiedene Aspekte exemplarisch ein Thema aufzugreifen, welches beispielhaft für ein bestimmtes Problem steht.

\section{Literatur}

Banse, Gerhard (2014): Unsicherheit und Ungewissheit als Normalität. Einige Gedanken nach dem nochmaligen Lesen von Günter Ropohls „Das Risiko im Prinzip Verantwortung“ (1994). In: Karafyllis, N.C. (Hg.): Das Leben führen? Lebensführung zwischen Technikphilosophie und Lebensphilosophie - Für Günter Ropohl zum 75. Geburtstag. Berlin: Leibniz Sozietät der Wissenschften, S. 221-237.

Bastow, Simon; Patrick Dunleavy; Jane Tinkler (2014): The impact of the social sciences: how academics and their research make a difference. London: Sage

Bateman, Scott; Mandryk, Regan L.; Gutwin, Carl; Genest, Aaron; McDine, David; Brooks, Christopher (2010): Useful junk?: the effects of visual embellishment on comprehension and memorability of charts. Proceedings of the SIGCHI Conference on Human Factors in Computing Systems, Atlanta/GA 2010, S. 2573-2582. (DOI:10.1145/1753326.1753716.)

Beck, Ulrich (1992): Risk society: Towards a new modernity. London: Sage.

Borkin, Michelle A.; Vo, Azalea A., Bylinskii, Zoya; Isola, Phillip; Sunkavalli, Shashank; Olivia, Aude; Pfister, Hanspeter (2013): What Makes a Visualization Memorable? IEEE Transactions on Visualization and Computer Graphics 12, 2306-15 (DOI: 1109/TVCG.2013.234).

Böschen, Stefan, Kastenhofer, Karen; Rust, Ina; Söntgen, Jens; Wehling, Peter (2008): Entscheidungen unter Bedingungen pluraler Nichtwissenskulturen. In: Mayntz, Renate; Neidhardt, Friedhelm; Weingart ,Peter; Wengenroth, Ulrich (Hg.): Wissensproduktion und Wissenstransfer. Wissen im Spannungsfeld von Wissenschaft, Politik und Öffentlichkeit. Bielefeld: Transcript, S. 197-220. 
Böschen, Stefan; Kastenhofer, Karen; Rust, Ina; Söntgen, Jens; Wehling, Peter (2010): Scientific Nonknowledge and Its Political Dynamics: The Cases of Agri-Biotechnology and Mobile Phoning. Science, Technology \& Human Values 35 (6), S. 783-811. DOI:10.1177/0162243909357911.

Böschen, Stefan; Kastenhofer, Luitgard Marschall, Karen; Rust, Ina; Söntgen, Jens; Peter Wehling (2006): Scientific cultures of non-knowledge in the controversy over genetically modified organisms (GMO). The Cases of Molecular Biology and Ecology. GAIA 15 (4), S. 294-301.

Brecht, Berthold (2004): Geschichten vom Herrn Keuner - Zürcher Fassung. Frankfurt am Main: Suhrkamp.

Christensen, Jon (2008): Smoking out objectivity: journalistic gears in the agnogenesis machine. In: Proctor, Robert N.; Schiebinger, Londa (eds.): Agnotology: The making and unmaking of ignorance. Stanford: Stanford University Press, S. 266-282.

Corbett, Julia B.; Durfee, Jessica L. (2004): Testing public (un) certainty of science media representations of global warming. Science Communication26 (2), S. 129-151. DOI:10.1177/1075547004270234.

De Marchi, Giada; Lucertini, Guilia; Tsoukiàs, Alexis (2016): From evidence-based policy making to policy analytics. Ann Oper Res 236 (1), S. 15-38. (DOI:10.1007/s10479-0141578-6.)

Dienel, Hans-Liudger; Meister, Martin (2006): Technik und Öffentlichkeit. Gemeinsame Jahrestagung der Gesellschaft für Technikgeschichte und der Gesellschaft für Wissenschafts- und Technikforschung. Technikgeschichte 73, S. 121-130.

Dienel, Hans-Liudger (2006): Schreiben, Zeichnen, Erinnern. Persönliches Wissensmanagement im Ingenieurberuf seit 1850. In: Rammert, Werner; Schubert, Cornelius (Hg.): Technografie. Zur Mikrosoziologie der Technik. Frankfurt am Main: Campus, S. 397-425.

Dienel, Hans-Liudger (2007): Anmerkungen $\mathrm{zu}$ einer Verfahrenswissenschaft für Realexperimente. In: Erwägen, Wissen, Ethik 18, S. 363-366.

Dienel, Hans-Liudger; Dierkes, Meinolf; Karlstetter, Elisabeth; Legewie, Heiner (2003): Von der Holschuld zur Bringschuld. Forschungswissen verfügbar machen durch das Forschungs-Informations-System Verkehr, Städtebau, Raumordnung und Wohnungswesen (FIS). In: Forum Qualitative Sozialforschung 4.

Dienel, Hans-Liudger; Sharan, Yair; Rapp, Christian; Ahituv, Niv (Hg.) (2010): Terrorism and the Internet. Threats, Target Groups, Deradicalisation Strategies. Amsterdam: IOS Press 2010.

Harrison, Lane; Reinecke, Katharina; Chang, Remco (2015) Infographic aesthetics: Designing for the first impression. Proceedings of the 33rd Annual ACM Conference on Human Factors in Computing Systems, S. 1187-1190. (DOI:10.1145/2702123.2702545.)

Hyder, Adnan A., Corluka, Adriana; Winch, Peter J. El-Shinnawy, Azza Harith; Hossein, Ghassany; Malekafzali; Lim, Meng-Kin; Mfutso-Bengo, Joseph; Segura, Elsa; Ghaffar, Abdul (2011): National policy-makers speak out: are researchers giving them what they need? Health Policy and Planning 26 (1), S. 73-82. (DOI: 10.1093/heapol/czq020).

Jensen, Jakob D. (2008): Scientific uncertainty in news coverage of cancer research: Effects of hedging on scientists and journalists credibility. Human communication research 34 (3) (2008), S. 347-369. (DOI: 10.1111/j.1468-2958.2008.00324.x.) 
Knorr-Cetina, Karin (1999) Epistemic cultures: How the sciences make knowledge. Cambridge: Cambridge University Press.

Lehmkuhl, Markus; Peters, Hans Peter (2016): Constructing (un-)certainty: An exploration of journalistic decision-making in the reporting of neuroscience. Public Understanding of Science 25 (8) (2016), S 909-926. (DOI:10.1177/0963662516646047.)

Majcen, Špela (2016): Evidence based policy making in the European Union: the role of the scientific community. Environmental Science and Pollution Research international 2016, S. 1-3. (DOI:10.1007/s11356-016-6247-7).

Merton, Robert. K. (1987): Three Fragments from a Sociologist s Notebooks: Establishing the Phenomenon, Specified Ignorance Strategic Research Materials. Annual Review of Sociology 13, S. 1-29.

Oreskes, Naomi; Conway, Erik M.; Shindell, Matthew (2008): From Chicken Little to Dr. Pangloss: William Nierenberg, Global Warming und the Social Deconstruction of Scientific Knowledge. Historical Studies in the Natural Sciences38 (1), S. 109-152.

Parascandola, Michael (2000): Health in the news: What happens when researchers and journalists collide. Research Practitioner 1, S. 1-29.

Pollack, Henry (2003): Uncertain Science ... Uncertain World. Cambridge: Cambridge University Press.

Poulos, Roslyn G.; Zwi, Anthony B. (2005): Letter to the Editor. Evidence-based policy making? Medical Journal of Australia 182 (8), S. 429.

Proctor, Robert N. (1995): Cancer Wars: How Politics Shapes What We Know and Don t Know about Cancer. New York: Basis Books.

Proctor, Robert N. (2008) Agnotology: A missing term to describe the cultural production of ignorance (and its study). In: Proctor, Robert N.; Schiebinger, Londa (eds.): Agnotology. The making and unmaking of ignorance. Stanford: Stanford University Press, S. 1-33.

Ravetz, Jerome R. (1987): Usable knowledge, usable ignorance: incomplete implications. In: Clark, William C.; Munn, Ralph E. (eds.): Sustainable development of the biosphere. Cambridge: Cambridge University Press, S. 415-32.

Schön, Susanne; Kruse, Sylvia; Meister, Martin; Nolting, Benjamin; Ohlhorst, Dorte (2007): Handbuch Konstellationsanalyse. Ein interdisziplinäres Brückenkonzept für die Nachhaltigkeits-, Technik- und Innovationsforschung. München: Oekom.

Simmerling, Anne; Nina Janich (2015): Rhetorical functions of a language ofuncertainty in the mass media. Public Understanding of Science 25 (8) (2015), 961-975. (DOI:10.1177/0963662515606681).

Singer, Eleanor; Endreny, Phyllis M. (1994): Reporting on Risk. How the Mass Media Portray Accidents, Diseases, Disasters and Other Hazards. RISK: Issues in Health \& Safetey 5, 261-270.

Smiciklas, Mark (2012): The Power of Infographics: Using Pictures to Communicate and Connect with Your Audience. Indianapolis: Que Publishing.

Smithson, Michael (1985): Toward a social theory of ignorance. Journal for the Theory of Social Behaviour15 (2), S. 151-72. DOI:10.1111/j.1468-5914.1985.tb00049.x.

Smithson, Michael (1989): Ignorance and uncertainty: Emerging paradigms. New York: Springer.

Stilgoe, Jack (2007): The (co-)production of public uncertainty: UK scientific advice on mobile phone health risks. Public Understanding of Science 16 (1), S. 45-61. 
(DOI:10.1177/0963662506059262).

Stocking, S. Holly; Holstein, Lisa W. (2008): Manufacturing doubt: journalists roles and the construction of ignorance in a scientific controversy. Public Understanding of Science 18 (1), S. 23-42. (DOI:10.1177/0963662507079373).

Tufte, Edward (1983): The Visual Display of Quantitative Information. Cheshire, Connecticut: Graphics Press.

Tufte, Edward (2001): The Visual Display of Quantitative Information (2nd ed.). Cheshire, Connecticut: Craphics Press.

Wehling, Peter (2006): Im Schatten des Wissens? Perspektiven der Soziologie des Nichtwissens. Konstanz: UVK.

Wehling, Peter (2009): Nichtwissen: Bestimmungen, Abgrenzungen, Bewertungen. Erwägen Wissen - Ethik 20 (1), S. 95-106.

Wehling, Peter (2015): Vom Nutzen des Nichtwissens: Sozial- und kulturwissenschaftliche Perspektiven. Bielefeld: Transkript.

Wehling, Peter; Böschen, Stefan (2015): Nichtwissenskulturen und Nichtwissensdiskurse. Über den Umgang mit Nichtwissen in Wissenschaft und Öffentlichkeit. Baden-Baden: Nomos.

Wilson, Kris M. (2000): Drought, debate, and uncertainty: Measuring reporters knowledge and ignorance about climate change. Public Understanding of Science 9 (1), S. 1-13. DOI:10.1088/0963-6625/9/1/301.

Zehr, Stephen C. (2000): Public representations of scientific uncertainty about global climate change. Public Understanding of Science 9 (2), S. 85-103.

DOI:10.1088/0963-6625/9/2/301.

\section{Angaben zu den Autoren}

Dienel, Hans-Liudger, Prof. Dr., Technische Universität Berlin, Fachgebiet Arbeitslehre, Technik und Partizipation, Marchstraße 23, 10587 Berlin, hans-liudger.dienel@tu-berlin.de

Henseler, Christoph, Technische Universität Berlin, Fachgebiet Arbeitslehre, Technik und Partizipation, Marchstraße 23, 10587 Berlin, christoph.henseler@tu-berlin.de 


\section{JOSHA}

Journal of Science, Humanities and Arts

January 2020

Volume 7, Issue 1 


\section{JOSHA}

Journal of Science, Humanities and Arts

January 2020

Volume 7, Issue 1 